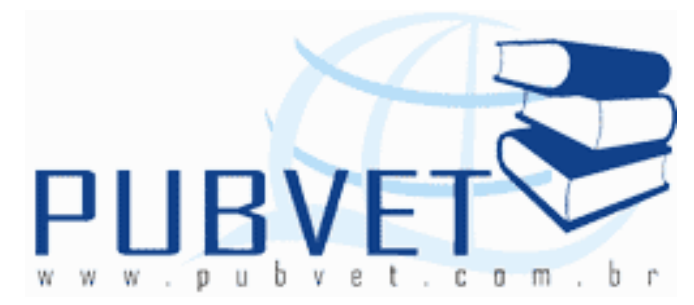

PUBVET, Publicações em Medicina Veterinária e Zootecnia.

\title{
Alterações hematológicas de cães naturalmente infectados por membros da família Anaplasmataceae diagnosticados pelo esfregaço de sangue periférico
}

\author{
Denner Santos dos Anjos ${ }^{1}$, Isabel Parizotto Metzdorf², Beatriz Candolo \\ Marques $^{2}$, Veronica Jorge Babo-Terra ${ }^{3}$
}

${ }^{1}$ Acadêmico do curso de Medicina Veterinária da FAMEZ / UFMS

2 Médicas Veterinárias Residentes da FAMEZ / UFMS

3Professora de Clínica Médica e Terapêutica de Pequenos Animais - FAMEZ / UFMS

\section{Resumo}

A erliquiose canina é uma doença infecciosa importante no Brasil, com maiores prevalências em regiões tropicais e subtropicais devido à distribuição do vetor. O objetivo do presente trabalho foi verificar as alterações hematológicas de cães infectados naturalmente por Ehrlichia spp. ou Anaplasma platys, diagnosticados pela visualização de mórulas em células mononucleares ou plaquetas no esfregaço sanguíneo. Foram avaliados 909 hemogramas durante o período de quatorze meses, de cães atendidos no Hospital Veterinário. Nas infecções por Ehrlichia spp., observou-se com maior frequência, anemia (74,85\%), eosinopenia $(62,85 \%)$, desvio nuclear de neutrófilos para a esquerda $(14,85 \%)$, linfopenia $(56,57 \%)$ e leucopenia $(29,71 \%)$. Nas infecções prováveis por Anaplasma platys observou-se, com maior frequência, linfopenia 
(51,85\%), leucopenia (24,07\%) e eosinopenia (44,44\%). Em ambas, em relação à morfologia, o tipo de anemia mais frequente foi normocítica normocrômica. Dessa maneira, eosinopenia, linfopenia, anemia normocítica normocrômica e leucopenia foram os achados mais comuns nas infecções estudadas.

Palavras-chave: cão, erliquiose, hematologia, mórulas

\title{
Hematological alterations of dogs naturally infected by members of Anaplasmataceae family diagnosed by peripheral blood smear
}

\begin{abstract}
Canine ehrlichiosis is a very important infectious disease in Brazil, with higher prevalence rates in tropical and subtropical regions due to the distribution of the vector. The aim of this study was to evaluate the hematological alterations of dogs naturally infected with Ehrlichia spp. or Anaplasma platys, diagnosed by the visualization of morulae in mononuclear cells or platelets in blood smears. We have evaluated 909 blood smears of dogs submitted to the Veterinary Hospital, during the period of fourteen months. In infections caused by Ehrlichia spp. the most frequent changes were anemia $(74.85 \%)$, eosinopenia $(62.85 \%)$, nuclear deviation of neutrophils to the left $(14,85 \%)$, lymphopenia (56.57\%) and leukopenia (29.71\%). On the other hand, in infections suggestive of Anaplasma platys it was observed lymphopenia (51.85\%), leukopenia (24.07\%) and eosinopenia (44.44\%). Among the anemic animals, the most common type of anemia was normocytic normochromic. Thus, eosinopenia, Iymphopenia, normocytic normochromic anemia and leukopenia were the most common findings in the studied infections.
\end{abstract}

Keywords: dogs, ehrlichiosis, hematology, morula 
ANJOS, D.S. et al. Alterações hematológicas de cães naturalmente infectados por membros da família Anaplasmataceae diagnosticados pelo esfregaço de sangue periférico. PUBVET, Londrina, V. 6, N. 20, Ed. 207, Art. 1386, 2012.

\section{Introdução}

Ehrlichia spp. são bactérias intracelulares obrigatórias, gram-negativas pertencentes à ordem Rickettsiales, família Anaplasmataceae (DUMLER,2001), que infectam várias espécies de mamíferos (VIEIRA et al., 2011). São transmitidas principalmente pelo carrapato Rhipicephalus sanguineus, com maior prevalência em regiões tropicais e subtropicais devido à presença do vetor (ANDEREG \& PASSOS, 1999). Vivem no interior da célula hospedeira, dentro de corpúsculos de inclusão, em estruturas denominadas mórulas (NYINDO et al.,1971) podendo ser vistas em monócitos, linfócitos (EWING, 1969; KUEHN \& GAUNT, 1985; ELIAS, 1991) e neutrófilos (EWING, 1969; HUXSOLL et al., 1970).

O primeiro relato de Ehrlichia canis no Brasil foi em Belo Horizonte (COSTA et al., 1973) e atualmente a doença encontra-se distribuída em todas as regiões do país, principalmente no sudeste e centro-oeste (ALMOSNY, 1998).

A infecção por Ehrlichia canis, responsável pela erliquiose monocítica canina, consiste em três fases: aguda, subclínica e crônica (NEER, 1998). Na fase aguda, as alterações laboratoriais englobam trombocitopenia associada ao aumento do número de macroplaquetas (SMITH et al., 1975); anemia arregenerativa ou regenerativa e contagens leucocitárias diversas. Leucocitose, monocitose e presença das mórulas são consideradas características (GREENE \& HARVEY, 1990; HARRUS et al., 1997).

$\mathrm{Na}$ fase subclínica, muitos animais tornam-se assintomáticos, permanecendo como portadores (HUXSOLL et al., 1970), podendo-se encontrar trombocitopenia, anemia, leucopenia variável, hiperglobulinemia progressiva e proteinúria (HARRUS et al., 1998). Na fase crônica observam-se pancitopenia, anemia aplásica, neutropenia e trombocitopenia com variados graus de supressão da série eritróide, mielóide e megacariocítica (WANER \& HARRUS, 2000; BREITSCHWERDT, 2004).

Pertencentes à mesma família Anaplasmataceae, Anaplasma platys é responsável pela trombocitopenia cíclica canina, infectando as plaquetas 
circulantes de cães, com formação de mórulas em seu interior (HARRUS et al., 1997; HUANG et al., 2005; LAPPIN, 2010). O carrapato $R$. sanguineus pode estar envolvido na transmissão do agente (SIMPSON et al.,1991). Cães infectados costumam apresentar anemia, trombocitopenia e leucocitose por neutrofilia (LAPPIN, 2010).

O diagnóstico é confirmado pela visualização das mórulas em exame de esfregaço sanguíneo, em monócitos e linfócitos circulantes, no caso da $E$. canis, ou em plaquetas, como ocorre na $A$. platys, em esfregaços sanguíneos corados pelo Giemsa, principalmente na fase aguda da infecção. Ambos os patógenos podem ser diagnosticados também por métodos moleculares como a Reação da Polimerase em Cadeia (PCR) (SOUSA, 2006).

$O$ objetivo do presente trabalho foi relatar as alterações hematológicas mais comuns encontradas em cães naturalmente infectados por Ehrlichia spp. ou Anaplasma spp, diagnosticados por meio da visualização de mórulas em esfregaços de sangue periférico.

\section{Material e Métodos}

Foram avaliados, no Laboratório de Patologia Clínica da Faculdade de Medicina Veterinária e Zootecnia (FAMEZ), 909 hemogramas de cães atendidos no período de março de 2010 a abril de 2011. Os animais foram submetidos à avaliação sanguínea - hemograma (eritrograma e leucograma) para auxílio diagnóstico ou exames de rotina. Os valores de referência para hemograma foram de acordo com Meinkoth \& Clinkenbeard (2000). Amostras de sangue foram coletadas por punção venosa e acondicionadas em tubos com anticoagulante (EDTA). Contagem diferencial dos leucócitos, avaliação da morfologia celular e pesquisa de hematozoários foram realizadas nos esfregaços sanguíneos corados pelo método May Grünwald - Giemsa e observadas em microscópio óptico, aumento de 100X. Cabe ressaltar que o método diagnóstico utilizado foi exclusivamente a visualização de corpúsculos de inclusão ou mórulas nas células sanguíneas. 
ANJOS, D.S. et al. Alterações hematológicas de cães naturalmente infectados por membros da família Anaplasmataceae diagnosticados pelo esfregaço de sangue periférico. PUBVET, Londrina, V. 6, N. 20, Ed. 207, Art. 1386, 2012.

\section{Resultados e Discussão}

Dos 909 hemogramas avaliados, 175 (19,25\%) apresentavam mórulas de Ehrlichia spp. e 54 (5,94\%) inclusões intraplaquetárias sugestivas de Anaplasma platys. Não foi possível identificar a espécie de Ehrlichia observada, pois não foram realizados exames de PCR. Entretanto, pode-se inferir que a espécie mais encontrada deve ter sido a E. canis, pois a grande maioria das mórulas foi detectada em mononucleares, além desta ser a espécie mais encontrada no Brasil. As alterações hematológicas dos cães infectados com Ehrlichia spp. e Anaplasma platys estão listadas nas Tabelas 1 e 2, respectivamente.

A maioria dos cães $(n=131)$ infectados por Ehrlichia spp. apresentou anemia $(74,85 \%)$ do tipo normocítica $(79,43 \%)$ normocrômica $(76,57 \%)$, corroborando com os achados de Kuehn \& Gaunt (1985), Accetta (2008), Borin et al. (2009) e Mendonça et al. (2005). A anemia pode ser devida à remoção dos eritrócitos circulantes pelo sistema monocítico-fagocitário, à ação do complemento na lise celular e à supressão da eritropoiese na medula óssea (MOREIRA et al., 2003). A hipoproteinemia e hiperproteinemia estiveram presentes em $17,14 \%$ e $29,14 \%$ dos cães, respectivamente, sendo que a maioria manteve-se dentro dos parâmetros normais (52,57\%). No leucograma, 29,71\% dos cães apresentaram leucopenia, 14,85\% leucocitose e $55,43 \%$ mantiveram-se dentro dos valores normais. A leucometria total não alterada corrobora com os achados de Mendonça et al. (2005), Accetta (2008) e Borin et al. (2009), os quais encontraram $61,47 \%$, $57,1 \%$ e $55,17 \%$, respectivamente. Entretanto, apesar da leucopenia ser a alteração mais relatada na infecção por Ehrlichia spp., o presente estudo encontrou 29,71\% dos cães leucopênicos, dados semelhantes aos de Mendonça et al. (2005) e Borin et al. (2009) que relataram a ocorrência em 24,70\% e 24,63\%, respectivamente, e inferiores aos de Waner et al. (1997), que relataram $78 \%$ de leucopenia. Os mecanismos responsáveis pela leucopenia podem ser os mesmos atribuídos à anemia, principalmente a hipoplasia da medula óssea (MENDONÇA et al., 2005). 
ANJOS, D.S. et al. Alterações hematológicas de cães naturalmente infectados por membros da família Anaplasmataceae diagnosticados pelo esfregaço de sangue periférico. PUBVET, Londrina, V. 6, N. 20, Ed. 207, Art. 1386, 2012.

$\mathrm{Na}$ contagem diferencial para leucócitos, foram observados $18,28 \%$ de neutropenia, $16,57 \%$ de neutrofilia, $42,28 \%$ de monocitose, $10,28 \%$ de basofilia, $56,57 \%$ de linfopenia e $62,85 \%$ de eosinopenia. Cento e dez cães apresentaram eosinopenia $(62,85 \%)$, condizendo com os achados de Waddle e Littman (1988), Mendonça et al. (2005) e Borin et al. (2009), os quais encontraram $63 \%, 64,22 \%$ e $58,13 \%$, respectivamente. O desvio nuclear de neutrófilos para a esquerda (DNNE) ocorreu em 14,85\% dos cães, resultados inferiores comparados a Moreira et al. (2003), Mendonça et al. (2005) e Borin et al. (2009), que encontraram $66,7 \%, 50,46 \%$ e $67 \%$, respectivamente. Contudo, esses valores foram superiores quando comparados com Accetta (2008), que observou $7,1 \%$ de DNNE. Este desvio se deve provavelmente à vasculite e focos de inflamação nos órgãos onde ocorre a multiplicação do agente, o que promove a migração, destruição e sequestro dos leucócitos (MENDONÇA et al., 2005).

A linfopenia observada em $56,57 \%$ dos cães do presente estudo é superior aos valores relatados por Waddle e Littman (1988), Mendonça et al. (2005), Accetta (2008) e Borin et al. (2009), que observaram 48\%, 22,02\%, $22,60 \%$ e 10,34\%, respectivamente. Estas alterações leucocitárias, eosinopenia e linfopenia são atribuídas à infecção aguda da doença, devido à ação dos corticosteróides e catecolaminas liberadas, provocando lise dos eosinófilos, linfólise e/ou seqüestro dos linfócitos nos tecidos corpóreos (MENDONÇA et al., 2005).

No caso dos cães que apresentaram mórulas em plaquetas, assumiu-se que se tratava de Anaplasma platys, pois não foi realizada a PCR. Nestes, a anemia esteve presente em $50 \%$ dos casos, sendo normocítica $(88,88 \%)$ normocrômica $(75,92 \%)$ em sua maioria. No leucograma, 59,26\% mantiveram-se dentro da faixa de normalidade, com $24,07 \%$ de leucopenia e $16,66 \%$ de leucocitose. Os animais apresentaram $51,85 \%$ de linfopenia, $37,03 \%$ de monocitose, $44,44 \%$ de eosinopenia e $14,81 \%$ de neutropenia. Devido à intensa leucopenia, não foi possível realizar o diferencial leucocitário em dois cães. 
ANJOS, D.S. et al. Alterações hematológicas de cães naturalmente infectados por membros da família Anaplasmataceae diagnosticados pelo esfregaço de sangue periférico. PUBVET, Londrina, V. 6, N. 20, Ed. 207, Art. 1386, 2012.

Tabela 1 Achados hematológicos de 175 cães domésticos naturalmente infectados por Ehrlichia spp., Campo Grande,MS.

\begin{tabular}{|c|c|c|c|c|}
\hline Parâmetros Avaliados & $\begin{array}{l}\text { Normal } \\
\text { n }(\%)\end{array}$ & $\begin{array}{l}\text { Diminuído } \\
\text { n (\%) }\end{array}$ & $\begin{array}{c}\text { Aumentado } \\
\text { n (\%) }\end{array}$ & $\begin{array}{l}\text { Valores de } \\
\text { Referência }\end{array}$ \\
\hline $\begin{array}{c}\text { Hematimetria } \\
\left(\times 10^{6} / \mu L\right)\end{array}$ & $39(22,28 \%)$ & $136(77,71 \%)$ & 0 & $5,5-8,5$ \\
\hline Hematócrito (\%) & $44(25,14 \%)$ & $131(74,85 \%)$ & 0 & $37-55$ \\
\hline Hemoglobina (g/dL) & $42(24 \%)$ & $133(76 \%)$ & 0 & $12-18$ \\
\hline VGM (fL) & $139(79,43 \%)$ & 0 & $36(20,57)$ & $60-77$ \\
\hline CHGM (g/dL) & $134(76,57 \%)$ & $34(19,43 \%)$ & $7(4 \%)$ & $32-26$ \\
\hline $\begin{array}{c}\text { Proteínas plasmáticas } \\
\text { totais }(\mathrm{g} / \mathrm{dL})\end{array}$ & $92(52,57 \%)$ & $51(29,14 \%)$ & $30(17,14 \%)$ & $6,0-8,0$ \\
\hline Reticulócitos $^{a}$ & $23(13,14 \%)$ & $46(26,28 \%)$ & $52(29,71 \%)$ & $0,5-1,0$ \\
\hline Indice Ictéricob & $150(85,71 \%)$ & 0 & $10(5,71 \%)$ & $2-5$ \\
\hline Leucócitos $^{c}\left(\times 10^{3} / \mu L\right)$ & $97(55,43 \%)$ & $52(29,71 \%)$ & $26(14,85 \%)$ & $6,0-17,0$ \\
\hline Bastões (/ $\mu L)$ & $144(82,28 \%)$ & 0 & $26(14,85 \%)$ & $0-300$ \\
\hline Segmentados (/ $\mu L)$ & $109(62,28 \%)$ & $32(18,28 \%)$ & $29(16,57 \%)$ & $3000-11500$ \\
\hline 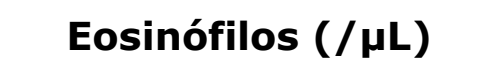 & $57(32,57 \%)$ & $110(62,85 \%)$ & $3(1,71 \%)$ & $100-1250$ \\
\hline Basófilos (/ $\mu L)$ & $152(86,85 \%)$ & 0 & $18(10,28 \%)$ & 0 \\
\hline Monócitos (/ $\mu \mathrm{L})$ & $92(52,57 \%)$ & $4(2,28 \%)$ & $74(42,28 \%)$ & $150-1350$ \\
\hline Linfócitos (/ $\mathbf{L L})$ & $70(40,0 \%)$ & $99(56,57 \%)$ & $1(0,57 \%)$ & $1000-4800$ \\
\hline
\end{tabular}

a- Não foi possível realizar a leitura de 54 amostras

b- 15 amostras estavam hemolisados

c- Devido à intensa leucopenia de cinco cães, não foi possível realizar o diferencial leucocitário. 
ANJOS, D.S. et al. Alterações hematológicas de cães naturalmente infectados por membros da família Anaplasmataceae diagnosticados pelo esfregaço de sangue periférico. PUBVET, Londrina, V. 6, N. 20, Ed. 207, Art. 1386, 2012.

Tabela 2 Achados hematológicos de 54 cães domésticos naturalmente infectados por Anaplasma platys, Campo Grande,MS.

\begin{tabular}{|c|c|c|c|c|}
\hline Parâmetros Avaliados & $\begin{array}{l}\text { Normal } \\
n(\%)\end{array}$ & $\begin{array}{c}\text { Diminuído } \\
n(\%)\end{array}$ & $\begin{array}{c}\text { Aumentado } \\
\text { n (\%) }\end{array}$ & $\begin{array}{l}\text { Valores de } \\
\text { Referência }\end{array}$ \\
\hline Hematimetria $\left(\times 10^{6} / \mu \mathrm{L}\right)$ & $23(42,59 \%)$ & $31(57,40 \%)$ & 0 & $5,5-8,5$ \\
\hline Hematócrito (\%) & $25(46,29 \%)$ & $29(53,70 \%)$ & 0 & $37-55$ \\
\hline Hemoglobina $(g / d L)$ & $26(48,15 \%)$ & $27(50,0 \%)$ & $1(1,85 \%)$ & $12-18$ \\
\hline VGM (fL) & $48(88,88 \%)$ & 0 & $6(11,11 \%)$ & $60-77$ \\
\hline CHGM (g/dL) & $41(75,92 \%)$ & $11(20,37 \%)$ & $2(3,70 \%)$ & $32-36$ \\
\hline $\begin{array}{c}\text { Proteínas plasmáticas } \\
\text { totais }(\mathrm{g} / \mathrm{dL})\end{array}$ & $29(53,70 \%)$ & $14(25,92 \%)$ & $11(20,37 \%)$ & $6,0-8,0$ \\
\hline Reticulócitos $^{\mathrm{a}}$ & $10(18,52 \%)$ & $10(18,52 \%)$ & $20(37,03 \%)$ & $0,5-1,0$ \\
\hline Indice Ictérico ${ }^{b}$ & $48(88,88 \%)$ & 0 & $1(1,85 \%)$ & $2-5$ \\
\hline Leucócitos $\left(\times 10^{3} / \mu L\right)$ & $32(59,26 \%)$ & $13(24,07 \%)$ & $9(16,66 \%)$ & $6,0-17,0$ \\
\hline Bastões $(/ \mu \mathrm{L})$ & $48(88,88 \%)$ & 0 & $4(7,40 \%)$ & $0-300$ \\
\hline Segmentados $(/ \mu L)$ & $37(68,52 \%)$ & $8(14,81 \%)$ & $7(12,96 \%)$ & $3000-11500$ \\
\hline Eosinófilos $(/ \mu \mathrm{L})$ & $22(40,74 \%)$ & $24(44,44 \%)$ & $6(11,11 \%)$ & $100-1250$ \\
\hline Basófilos $(/ \mu \mathrm{L})$ & $45(83,33 \%)$ & 0 & $7(12,96 \%)$ & 0 \\
\hline Monócitos (/ $\mu \mathrm{L})$ & $30(55,55 \%)$ & $2(3,70 \%)$ & $20(37,03 \%)$ & $150-1350$ \\
\hline Linfócitos (/ $\mu \mathrm{L})$ & $23(42,59 \%)$ & $28(51,85 \%)$ & $1(1,85 \%)$ & $1000-4800$ \\
\hline
\end{tabular}

a-não foi possível a realização da contagem de reticulócitos de14 cães.

b-cinco amostras estavam hemolisadas, não sendo avaliado o índice ictérico. 
ANJOS, D.S. et al. Alterações hematológicas de cães naturalmente infectados por membros da família Anaplasmataceae diagnosticados pelo esfregaço de sangue periférico. PUBVET, Londrina, V. 6, N. 20, Ed. 207, Art. 1386, 2012.

Infelizmente não foi realizada a contagem de plaquetas, e apesar da trombocitopenia ser descrita como o principal achado hematológico em cães infectados por $E$. canis e $A$. platys (ACCETTA, 2008), a observação de plaquetas nos esfregaços forneceu uma estimativa preditiva da plaquetometria, que em geral não parecia estar diminuída.

\section{Conclusão}

Pode-se observar que anemia normocítica normocrômica, hiperproteinemia, desvio nuclear de neutrófilos para a esquerda, eosinopenia e linfopenia foram achados frequentes na erliquiose e anaplasmose canina.

\section{Referências}

ACCETTA, E.M.T. Ehrlichia canis e Anaplasma platys em cães (Canis familiaris, Linnaeus, 1758) trombocitopênicos da região dos lagos do Rio de Janeiro. Tese de Mestrado. Rio de Janeiro: Universidade Federal Rural do Rio de Janeiro, Curso de Pós-Graduação em Medicina Veterinária, 2008.

ALMOSNY, N.R.P. Ehrlichia canis ( Donatien \& Lestoquard, 1935): Avaliação parasitológica, hematológica e bioquímica sérica da fase aguda de cães e gatos experimentalmente infectados. Tese de Doutorado. U.F.R.R.J. Itaguaí- RJ, 1998.

ANDEREG, P.I.; PASSOS, L.M.F. Erliquiose canina: revisão. Clínica Veterinária, v.4, n.19, p. 31-38, 1999.

BORIN, S.; CRIVELENTI, L.Z.; FERREIRA, F.A. Aspectos epidemiológicos, clínicos e hematológicos de 251 cães portadores de mórula de Ehrlichia spp. naturalmente infectados. Arquivo Brasileiro de Medicina Veterinária e Zootecnia, v.61, n.3, p. 566-571, 2009.

BREITSCHWERDT, E.B. Riquetsioses In: ETTINGER, S.J.; FELDMAN, E.C. Tratado de Medicina Interna Veterinária. Doenças do Cão e do Gato. $5^{\circ}$ ed. Rio de Janeiro: Guanabara Koogan, p. 422-429, 2004.

COSTA, J. O., SILVA, M., GUIMARÃES, M. P., BATISTA JUNIOR, J. Ehrlichia canis infection in a dog in Belo Horizonte- Brazil. Arquivos Escola Veterinária UFMG, v. 25, p. 199-200, 1973.

DUMLER, J.S.; BARBET, A.F.; BEKKER, C.P.J.; DASCH, G.A.; PALMER, G.H.; RAY, S.C.; RIKIHISA, Y.; RURANGIRWA, F. R. Reorganization of genera in the families Rickettsiaceae and Anaplasmataceae in the order Rickettsiales: unification of some species of Ehrlichia with Anaplasma, Cowdria with Ehrlichia and Ehrlichia with Neorickettsia, descriptions of six new species combinations and designation of Ehrlichia equi and 'HGE agent' as subjective synonyms of Ehrlichia phagocytophila. International Journal of Systematic and Evolutionary Microbiology, v. 51, n.6, p.2145-2165, 2001.

ELIAS, E. Diagnosis of ehrlichiosis from the presence of inclusions bodies or morulae of $E$. canis. Journal of Small Animal Practice, v.3, n.1, p.540-543, 1991. 
EWING, S. A. Canine ehrlichiosis. In: BRANDLY, C. A., CORNELIUS, C. E. Advances in Veterinary Science and Comparative Medicine. New York: Academic Press, v.13, p.331$353,1969$.

GREENE, C.E.; HARVEY, J.W. Canine ehrlichiosis. In: CE Greene, Infectious Diseases of the Dog and Cat, WB Saunders, Philadelphia, p. 545-561, 1990.

HARRUS, S.; BARK, H.; WANER, T. Canine monocytic ehrlichiosis: An update. The Compendium Continuing Education, v. 19, n.4, p. 431-444, 1997.

HARRUS, S.; WANER, T.; AIZENBERG, I.; FOLEY, J.E.; POLAND, A.M.; BARK, H. Amplification of ehrlichial DNA from dogs 34 months after infection with Ehrlichia canis. Journal of Clinical Microbiology, v. 36, p. 73-76, 1998.

HARVEY, J.W.; SIMPSON, C.F.; GASKIN, J.M. Cyclic thrombocytopenia induced by a Rickettsia like agent in dogs. Journal of Infectious Diseases, v.137, p.182-188, 1978.

HUANG, H.; UNVER, A.; PEREZ, M.J.; ORELLANA, N.G.; RIKIHISA, Y. Prevalence and molecular analysis of Anaplasma platys in dogs in Lara, Venezuela. Brazilian Journal of Microbiology, v.36, p.211-216, 2005.

HUXSOLL, D. L., HILDEBRANDT, P. K., NIMS, R. M., WALKER, J. S. Tropical canine pancytopenia. Journal of the American Veterinary Medical Association, v.157, n.1, p.1627-1632, 1970.

KUEHN, N.F.; GAUNT, S.D. Clinical and hematologic findings in canine ehrlichiosis. Journal of the American Veterinary Medical Association, v.186, p. 355-358, 1985.

LAPPIN, M.R. Doenças Infecciosas. In: NELSON, R.W.; COUTO, C.G. Medicina Interna de Pequenos Animais, 40ed. Rio de Janeiro: Elsevier, p. 1324-1325, 2010.

MEINKOTH, J.H.; CLINKENBEARD, K.D. Normal hematology of the dog. In: FELDMAN, B.F.; ZINKL, J.G.; JAIN, N.C. Schalm's veterinary hematology. $\mathbf{5}^{\circ} \mathbf{e d}$. Lippincott Williams \& Wilkins, p.787, 2000.

MENDONÇA, C.S.; MUNDIM, A.V.; COSTA, A.S.; MORO, T.V. Erliquiose canina: Alterações hematológicas em cães domésticos naturalmente infectados. Bioscience Journal, v. 21, n.1, p.167-174, 2005.

MOREIRA, S.M.; BASTOS, C.V.; ARAÚJO, R.B.; SANTOS, M.; PASSOS, L.M.F. Estudo retrospectivo (1998 a 2001) da erliquiose canina em Belo Horizonte. Arquivos Brasileiros de Medicina Veterinária e Zootecnia, v.55, p.141-147, 2003.

NEER, T. M. Canine monocytic granulocytic ehrlichiosis. In: GREENE, C. E. (Ed). Infectious Disease of the Dog and Cat. 2nd ed. Philadelphia: W. B. Saunders, p. 139-147, 1998.

NYINDO, M.B.A.; RISTIC, M.; HUXSOLL, D.L.; SMITH, A.R. Tropical canine pancytopenia: In vitro cultivation of the causative agent- Ehrlichia canis. American Journal of Veterrinary Research, v.32, n.11, p. 1651-1658, 1971.

SIMPSON, R.M.; GAUNT, S.D.; HAIR, J.A.; KOCAN, K.M.; HENK, W.G.; CASEY, H.W. Evaluation of Rhipicephalus sanguineus as a potential biologic vector of Ehrlichia platys. American Journal Veterinary Research, v. 52, n.9, p. 1537-1541, 1991. 
SMITH, R.D.; RISTIC, M.; HUXSOLL, D.L.; BAYLOR, R.A. Platelet kinetics in canine ehrlichiosis: evidence for increased platelet destruction as the cause of thrombocytopenia. Infection and Immunity, v. 11, p.1216-1221, 1975.

SOUSA, V.R.F. Avaliação clínica, morfológica, hematológica, bioquímica e biomolecular de cães naturalmente infectados por Ehrlichia canis e Anaplasma platys. Tese de Doutorado. Rio de Janeiro: Universidade Federal Rural do Rio de Janeiro; 2006.

VIEIRA, R.F.C.; BIONDO, A.W.; GUIMARÃES, A.M.S.; SANTOS, A.P.; SANTOS, R.P.; DUTRA, L.H. et al. Ehrlichiosis in Brazil. Revista Brasileira de Parasitologia Veterinária, v.20, p.01-12, 2011.

WADDLE, J.R.; LITTMAN, M.P. A retrospective study of 27 cases of naturally occurring canine ehrlichiosis. Journal of the American Animal Hospital Association, v.24, p.615-620, 1988.

WANER, T.; HARRUS, S.; BARK, H.; BOGIN, E.; AVIDAR, Y.; KEYSARY, A. Characterization of the subclinical phase of canine ehrlichiosis in experimentally infected beagle dogs. Veterinary Parasitology, v.69, p. 307-317, 1997.

WANER, T.; HARRUS, S.; Canine monocytic ehrlichiosis (CME). In: Recent Advances in Canine Infectious Disease. Disponível em: <http://www.ivis.org>, p. 01-05, 2000. 